\title{
Density Functional Theory and Tight Binding-Based Dynamical Studies of Carbon-Metal Systems of Relevance to Carbon Nanotube Growth
}

\author{
Kim Bolton ${ }^{1}(\varangle)$, Anders Börjesson ${ }^{1,2}$, Wuming Zhu², Hakim Amara ${ }^{3}$, and Christophe Bichara ${ }^{4}$ \\ ${ }^{1}$ School of Engineering, University of Borås, SE-501 90, Borås, Sweden \\ ${ }^{2}$ Department of Physics, Gothenburg University, SE-412 96, Gothenburg, Sweden \\ ${ }^{3}$ LEM, ONERA/CNRS, 29 Avenue de la Division Leclerc, F92322 Chatillon, France \\ ${ }^{4}$ CINaM, CNRS, 13288 Marseille, France \\ Received: 3 April 2009 / Revised: 25 May 2009 / Accepted: 25 May 2009 \\ CTsinghua University Press and Springer-Verlag 2009. This article is published with open access at Springerlink.com
}

\begin{abstract}
Density functional theory (DFT) and tight binding (TB) models have been used to study systems containing single-walled carbon nanotubes (SWNTs) and metal clusters that are of relevance to SWNT growth and regrowth. In particular, TB-based Monte Carlo (TBMC) simulations at 1000 or $1500 \mathrm{~K}$ show that Ni atoms that are initially on the surface of the SWNT or that are clustered near the SWNT end diffuse to the nanotube end so that virtually none of the $\mathrm{Ni}$ atoms are located inside the nanotube. This occurs, in part, due to the lowering of the Ni atom energies when they retract from the SWNT to the interior of the cluster. Aggregation of the atoms at the SWNT end does not change the chirality within the simulation time, which supports the application of SWNT regrowth (seeded growth) as a potential route for chirality-controlled SWNT production. DFT-based geometry optimisation and direct dynamics at $2000 \mathrm{~K}$ show that $\mathrm{Cr}$ and $\mathrm{Mo}$ atoms in $\mathrm{Cr}_{5} \mathrm{Co}_{50}$ and $\mathrm{Mo}_{5} \mathrm{Co}_{50}$ clusters prefer to be distributed in the interior of the clusters. Extension of these calculations should deepen our understanding of the role of the various alloy components in SWNT growth.
\end{abstract}

\section{KEYWORDS}

Carbon nanotube growth, metal alloy clusters, tight binding, Monte Carlo, direct dynamics

\section{Introduction}

One of the major driving forces behind the immense amount of research into single-walled carbon nanotubes (SWNTs) is their potential use in electronic devices [1, 2]. Depending on their chirality, SWNTs are either metallic or semiconducting and can therefore be used as interconnects or in transistors. However, it is not yet possible to grow metallic SWNTs in the absence of semiconducting nanotubes, or vice versa. The ultimate goal would, in fact, be to grow an SWNT with a desired chirality in the absence of other chiralities, preferably at the desired place in the electronic circuit.

Two methods that have shown potential for selective chiral growth are rapid heating based on cobalt-molybdenum (Co:Mo) catalysts [3] and SWNT regrowth [4] (also called seeded growth). In the former method the alloy particles are rapidly heated while carbon feedstock is introduced for SWNT

Address correspondence to kim.bolton@hb.se 
growth. It is thought that the rapid heating and the presence of the Mo prevent extensive coalescence of the Co particles, which subsequently grow SWNTs with diameter $\sim 0.8 \mathrm{~nm}$. The absence of large clusters prevents growth of large diameter SWNTs and thinner SWNTs are not stable due to their high curvature energy. This method also favours the growth of SWNTs that have near-armchair chiralities (e.g., $(6,5)$ nanotubes) [3].

In the second method [4], SWNTs are used as seeds to grow longer SWNTs. SWNTs are purified and carefully cut (to expose open SWNT ends) before metal atoms are evaporated into the reaction chamber. The chamber is subsequently heated and feedstock is introduced. The metal atoms form clusters (probably of various sizes and locations in the chamber) that aggregate at the SWNT end. These clusters catalyse regrowth at the end of the SWNT seed. Although it has been shown [4] that the distribution in seed chiralities affects those of the regrown SWNTs, it is not known if chirality is controlled at the individual SWNT level.

Experimental studies of SWNT growth are complemented by computational modelling, which offers control and analysis at the atomic level. Previous calculations include density functional theory (DFT) geometry optimisation [5, 6], molecular dynamics [7, 8], direct dynamics based on tight binding [9] and DFT [10,11] forces, and tight binding Monte Carlo (TBMC) simulations [1214]. For example, previous TBMC simulations at $1000 \mathrm{~K}$ showed that $\mathrm{Ni}$ atoms that are uniformly distributed over an SWNT wall aggregate and diffuse to the open end of the SWNT, where they remain for the remainder of the simulation [14]. This is important to SWNT regrowth where, as discussed above, one evaporates metal atoms into the growth chamber before heating and introducing feedstock for regrowth [4]. In this contribution we present results when the $\mathrm{Ni}$ atoms are not initially uniformly distributed over the SWNT surface, which is relevant to the regrowth experiments since heating of the system is expected to lead to aggregation of clusters at different places in the chamber, e.g., on the SWNT walls, before they diffuse to the SWNT end.

Our TBMC simulations are limited to carbon systems attached to a single metal, i.e., $\mathrm{Ni}$, since the semiempirical parameters have not been fitted for other metals [13]. However, many experimental growth methods use metal alloys such as the Co:Mo [3] alloy mentioned above. A detailed understanding of the growth mechanism on these alloy catalysts requires knowledge of the cluster structure during growth. For example, it is not known if the Co and Mo are homogenously mixed during growth, or if one of these metals forms a surface layer on the cluster. It is also not known if the presence of carbon feedstock species and the carbon nanotube (CNT) affects the distribution of the cluster components, e.g., if the nanotube preferentially bonds to one of the metals. Here we use forces obtained from DFT to study the structure of Co:Mo and Co:Cr alloy structures at $0 \mathrm{~K}$ and $2000 \mathrm{~K}$ (i.e., we perform direct dynamics simulations based on DFT forces). In addition to obtaining knowledge of the alloy structures at high temperatures, we also investigate whether calculations at $0 \mathrm{~K}$, which are computationally far cheaper than direct dynamics, provide information about the alloy cluster composition that is relevant to the high temperatures used in CNT growth. Since the Co:Mo and Co:Cr clusters show very similar compositions at the temperatures investigated, and since we have studied the latter system in more detail, we limit our presentation to $\mathrm{Co:Cr}$ clusters.

\section{Methods}

Simulations of the SWNT-Ni systems are based on a fourth moment TB approximation [12] combined with canonical MC [15]. This was used to study these systems at 1000 or $1500 \mathrm{~K}$ and provides good statistics for a variety of SWNT chiralities and initial distributions of the $\mathrm{Ni}$ atoms. This TB Hamiltonian has been developed specifically for nickel-carbon systems [13], and has been successfully used by Amara et al. [12] to study the formation of graphitic carbon structures on Ni surfaces and clusters. Their study showed that solubility of carbon atoms in nickel is limited, but that carbon atoms are also stable at subsurface sites. The validity of the Hamiltonian for studying SWNT-Ni cluster systems has been confirmed by comparison with DFT calculations [14]. 
As exemplified below, various initial structures are chosen for the simulations. In all cases the $\mathrm{Ni}$ atoms are initially located on the SWNT wall (both interior and exterior to the SWNT) or near an SWNT open end. Results when the atoms are initially uniformly distributed on the walls have been presented elsewhere [14], and here we present results when the atoms are initially clustered on the SWNT wall.

Two types of initial configurations were used when the atoms were initially clustered near the SWNT end. In the first type, previously called a gently docked structure [14], the cluster is placed below the lowest SWNT ring, and in the second type, previously called forced docking, the SWNT is immersed $\sim 1.5 \AA$ into the cluster. The initial Ni clusters are cut from the bulk nickel face centered cubic (fcc) structure. The structure of the metal cluster was relaxed before starting the MC simulations.

Long ( 30 A) SWNTs were used in the TB calculations, and a wide variety of chiralities were investigated. These SWNTs are longer than the TB cutoff distance, and the similarity between the results obtained from these SWNTs and those obtained from shorter capped SWNTs [14] supports the fact that the use of long or capped SWNTs does not affect the data presented here. The calculations were performed at 1000 or $1500 \mathrm{~K}$, which is relevant to chemical vapor deposition growth and regrowth of SWNTs. All data presented here are obtained after performing a minimum of $60,000 \times$ $N$ MC displacements, where $\mathrm{N}$ is the number of unconstrained atoms, to ensure equilibration (seen by constant average properties).

The total energy DFT calculations were performed using the Vienna ab initio simulation package (VASP) [16]. The geometry optimisation studies were performed using both the generalised gradient approximation (GGA) and local density approximation (LDA). In the former case the electron exchange-correlation energy was approximated by the Perdew-Wang functional (PW91) [17] and the in latter case the Perdew-Zunger (PZ) [18, 19] functional was used. Core electrons were treated using ultrasoft pseudopotentials. The cutoff energy of the planewave basis set was $236.5 \mathrm{eV}$ and the supercells were sufficiently large to allow for single $\Gamma$-point sampling. Spin-polarisation was used for the GGA calculations and both spin polarised and non spin polarised results were obtained for the LDA method. Structures were optimised using a conjugate-gradient (CG) algorithm without symmetry constraint, and total energies were converged to less than $1 \mathrm{meV}$.

Due to computational limitations and due to the similarity of the GGA and LDA results, the direct dynamics simulations were based on LDA non spin polarised forces. The integration time step was $1 \mathrm{fs}$, and the temperature was set to $2000 \mathrm{~K}$ to accelerate the equilibration rate. A constant temperature was maintained by scaling the velocities at each trajectory step. Typical initial cluster structures are discussed below, and the trajectories are typically $\sim 4$ ps in length.

\section{Results and discussion}

As discussed above, the distribution of metal atoms during regrowth experiments, i.e., during evaporation and heating, cannot be precisely controlled. We therefore simulate systems where the $\mathrm{Ni}$ atoms are not initially uniformly distributed over the SWNT surface, and compare the results to those when the atoms are initially uniformly distributed (which are reported elsewhere [14]). Figure 1 shows an example of the initial (Fig. 1(a)) and final (Fig. 1(b)) SWNT-

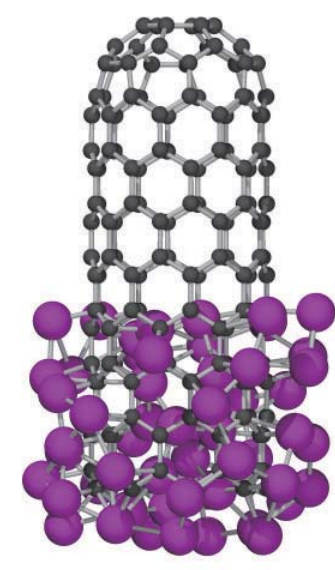

(a)

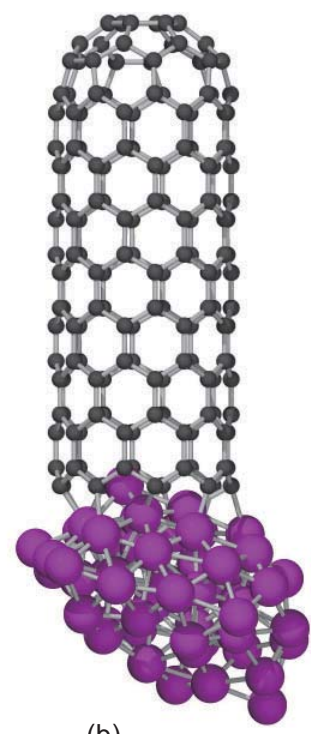

(b)
Figure 1 An example of the initial (a) and final (b) SWNT-Ni ${ }_{55}$ structures studied using TBMC 
metal structures studied here. It is clear that the $\mathrm{Ni}$ atoms are not initially uniformly distributed over the SWNT surface, but are grouped near the open end of the SWNT. All atoms move to the SWNT end, and remain there for the remainder of the MC simulation. These results are independent of how the Ni atoms are initially placed on the SWNT surface.

Figure 2 shows the local energy distributions of the carbon (Fig. 2(a)) and nickel (Fig. 2(b)) atoms corresponding to the structures shown in Fig. 1. The local energies of the $\mathrm{Ni}$ atoms decrease due to their diffusion and clustering, and hence Ni contributes to the lowering in the enthalpy (and Gibbs free energy). The average energy of the carbon atoms before and after clustering is $-7.2 \mathrm{eV}$, and for $\mathrm{Ni}$ it changes from -3.1 to $-3.5 \mathrm{eV}$. The contribution of the $\mathrm{Ni}$ to the change in Gibbs free energy is expected to be reduced due to a decrease in entropy that results from the clustering of the $\mathrm{Ni}$ atoms.

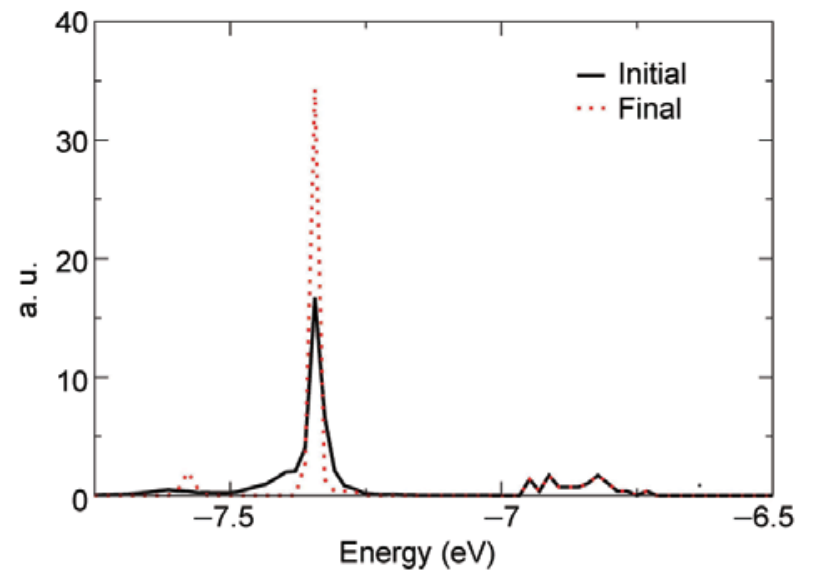

(a)

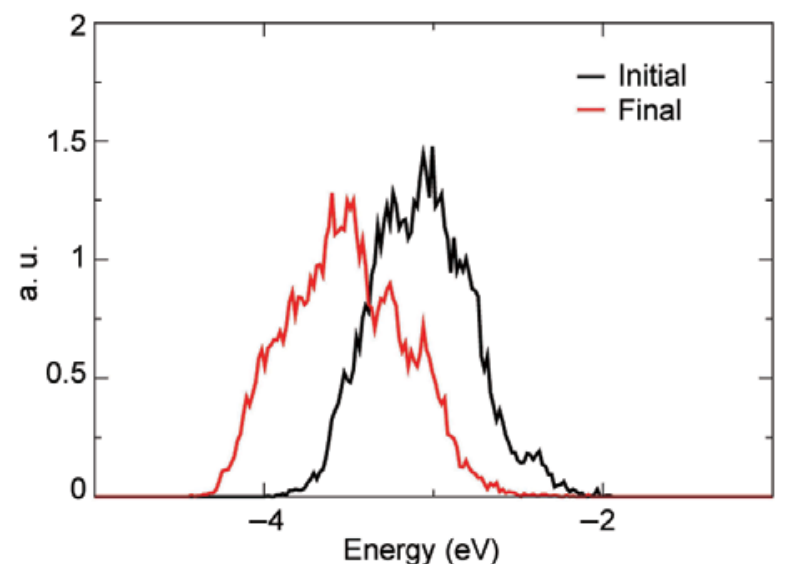

(b)

Figure 2 Local energy distributions of the carbon (a) and nickel (b) atoms corresponding to the structures shown in Fig. 1
The simulations discussed above were complemented by studying the changes in SWNTcluster geometries and energies when the cluster is initially placed slightly inside the SWNT (called gentle docking [14]) and when the open end of the SWNT is initially $\sim 1.5 \AA$ below the cluster surface (called forced docking [14]). Penetration of the metal cluster into carbon nanotubes, similar to the gently docked structures, has been observed during growth and the high-energy structures obtained by forced docking may be relevant to SWNT-electrode interfaces when the metal electrode is sputtered onto the SWNT interconnects.

Figures 3(a) and 3(b) show the initial and final structures when the $\mathrm{Ni}_{55}$ cluster is gently docked onto $(5,5)$ and $(10,0)$ SWNTs, and Fig. 3(c) shows the final structures for $(8,3)$ and $(10,1)$ SWNTs. As illustrated in the figure, and as is observed for all SWNT chiralities that we have simulated, the cluster moves from the SWNT interior to the SWNT open end, such that virtually all $\mathrm{Ni}$ atoms are located outside the nanotube. This is also observed when performing 0 K DFT geometry optimisations [14], confirming the validity of the TBMC results. Retraction of $\mathrm{Ni}$ atoms from the SWNT interior to the exterior of the SWNT end is driven by the lowering in energy of the $\mathrm{Ni}$ atoms. That is, the $\mathrm{Ni}$ atoms that are initially inside the SWNT have lower energy than when they are in the cluster. In fact, $\mathrm{Ni}$ atoms on the surface of the cluster have higher energies than the $\mathrm{Ni}$ atoms that are inside the SWNT [14], so retraction will only occur if the atoms that are inside the SWNT do not lead to a large increase in the cluster surface area. This is in agreement with previous studies [20], and means that clusters below a critical size will not retract from SWNTs. Similarly, clusters above this critical size will not, under equilibrium conditions, penetrate into carbon nanotubes. This, in turn, indicates that the penetration of large metal clusters into carbon nanotubes that is observed under experimental growth conditions is driven by other factors than those discussed here, such as a strong attraction between the cluster and defects in the nanotube.

Figures 4(a)-(c) show the final structures after forced docking of the $\mathrm{Ni}_{55}$ cluster on the $(5,5),(10,0)$,

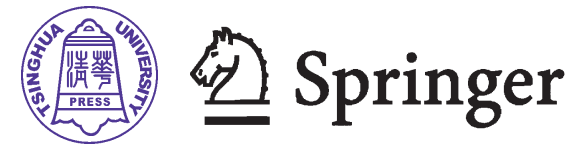


$(5,5)$

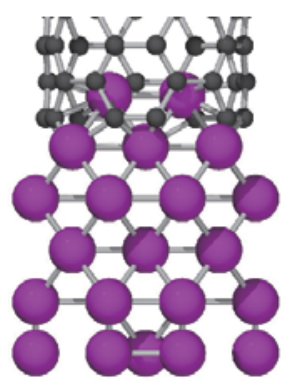

$(5,5)$

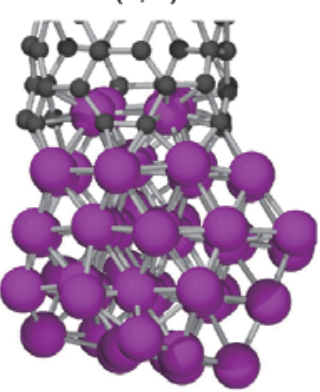

(b)

$(8,3)$

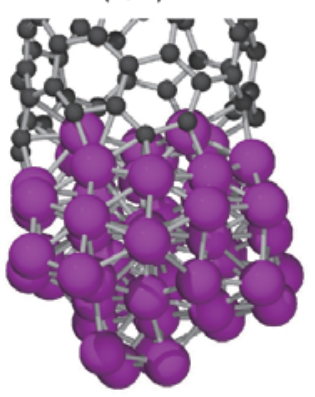

(a)
$(10,0)$
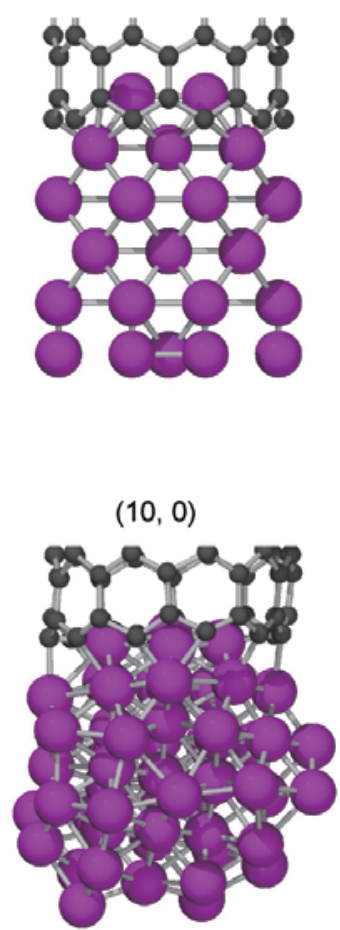

$(10,1)$

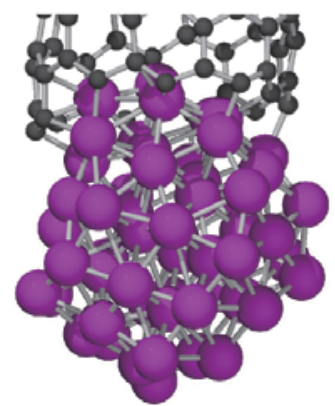

(c)

Figure 3 Initial (a) and final (b) structures when the $\mathrm{Ni}_{55}$ cluster is gently docked onto $(5,5)$ and $(10,0)$ SWNT. The final structures for $(8,3)$ and $(10,1)$ SWNTs are shown in (c) and $(8,3)$ SWNTs, respectively. Similarly to the results obtained from the gentle docking, the cluster moves to the outside of the SWNT open end. However, in contrast to the results obtained from the gentle docking, carbon atoms that are initially part of the SWNT dissolve into the cluster within the simulation time. Retraction of the cluster and dissolution of the SWNT end atoms was investigated for the $(10,0)$ nanotube by monitoring the local atomic energies. Comparison of the energy distributions before and after retraction and dissolution show that the energies of the two carbon atoms that dissolved into the cluster decreased from $-5.8 \mathrm{eV}$ to -6.5 and $-6.9 \mathrm{eV}$. However, the dissolution of these two atoms means that three other SWNT atoms, which were initially in the upper ring in Fig. 4(b) and that have three carbon neighbours, now have two carbon neighbours (i.e., they are edge atoms). The energies of these three atoms increase from $-7.2 \mathrm{eV}$ to $-5.8 \mathrm{eV}$. Hence, the change in the local energies of the carbon atoms disfavours retraction and dissolution. (In contrast, the increase in the entropy due to dissolution favours this process). Retraction and dissolution lead to a decrease in local energy distributions of the $\mathrm{Ni}$ atoms which favours this process.

It may be noted that dissolution of carbon atoms from $(n, 0)$ zigzag SWNTs that contain a complete end ring of $n / 2$ atoms, such as that described above for the $(10,0)$ SWNT, leads to the formation of even more end atoms (e.g., dissolution of the two atoms described above led to the formation of three new end atoms). This is unique for zigzag nanotubes, since it is possible to remove end atoms from other

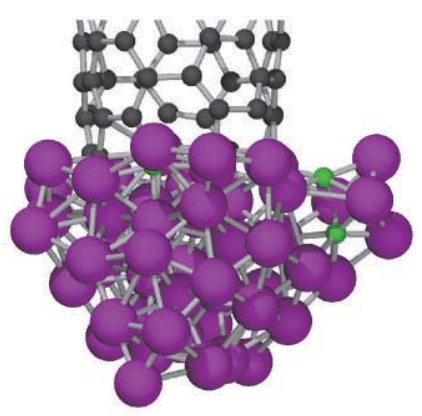

(a)

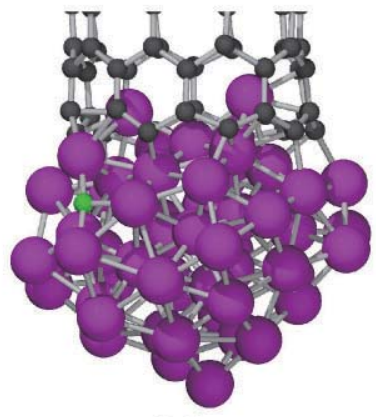

(b)

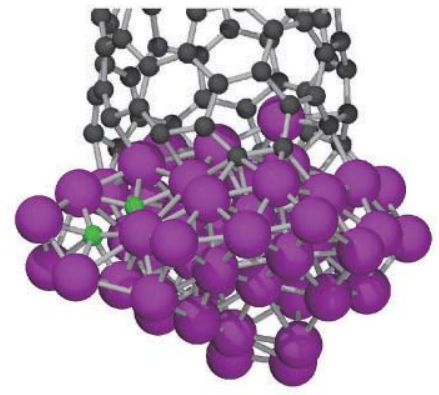

(c)

Figure 4 Final structures after forced docking of the $\mathrm{Ni}_{55}$ cluster on the $(\mathrm{a})(5,5),(\mathrm{b})(10,0)$, and $(c)(8,3)$ SWNTs. Dissolved carbon atoms are shown in green 
nanotube chiralities without increasing the number of end atoms. This should play an important role in the ease with which SWNTs (or walls within multiwalled carbon nanotubes) can dissolve into metals, and may also affect growth mechanisms and rates.

It may also be noted that the gently docked structures do not typically lead to dissolution of SWNT carbon atoms within our MC simulation time, whereas all of the forced docked structures lead to dissolution. In addition, preliminary TBMC results indicate that the structures with dissolved carbon (obtained from forced docking) may have lower energies than those with no dissolved carbon (obtained from gentle docking). This suggests that carbon dissolution from gentle docked structures is prevented by an activation barrier. It is not yet evident whether this barrier is sufficiently large to be relevant to experimental SWNT regrowth conditions and time. We are presently performing additional studies to ascertain the dissolution mechanism and rate.

Due to the parameters that are available in our TB model, the above discussion is limited to Ni catalysts. However, since most experiments are performed with alloy catalysts, we extend the above studies to bi-metallic catalysts. As discussed above, we report results of the Cr:Co alloy since this is the system that has been the focus of our studies. Simulations of Mo: Co catalysts, which are commonly used for SWNT growth, show that they have similar behaviour.

Figure 5 is a cross-section of a $\mathrm{CrCO}_{54}$ cluster (i.e., containing $1 \mathrm{Cr}$ atom and $54 \mathrm{Co}$ atoms), illustrating the centre, subsurface and outer layers. The structure

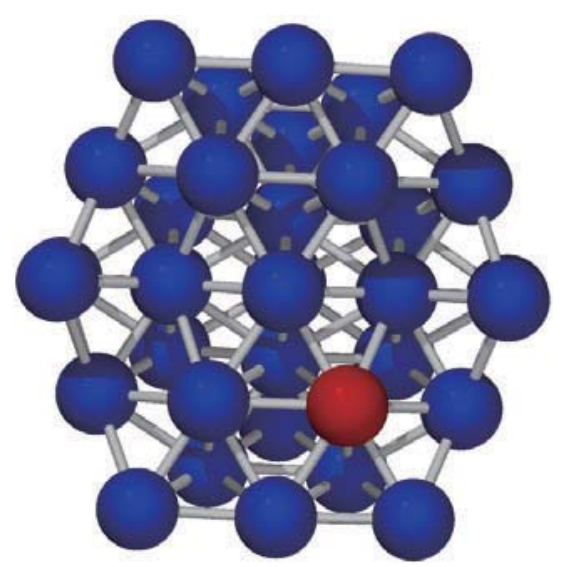

Figure 5 Cross-section of a $\mathrm{CrCO}_{54}$ cluster showing the center, subsurface and outer layers. The $\mathrm{Cr}$ atom is in the subsurface layer is that obtained from the GGA spin polarised calculations, and shows the $\mathrm{Cr}$ atom in the subsurface layer of the cluster. The energy of this structure is $-334.3 \mathrm{eV}$. Table 1 lists the energies obtained for this structure, when the $\mathrm{Cr}$ atom is at an apex or edge site in the outer layer, and when it is the centre atom.

Table 1 reveals that the four different cluster structures have similar energies, irrespective of the whether GGA, LDA or spin polarisation is used. Although the structures where the $\mathrm{Cr}$ atom occupies the central or subsurface positions are slightly preferred, the difference between the energy of this structure and the least preferred structure is less than $0.6 \mathrm{eV}$ for GGA and $1.3 \mathrm{eV}$ for the LDA methods. Also, although the structure with the highest energy depends on the computational method (subsurface site for GGA and surface sites for LDA methods), the energy differences between the different sites (for a given computational method) are very small. Hence, these $0 \mathrm{~K}$ calculations based on a single $\mathrm{Cr}$ atom indicate that this atom has a weak preference to be in the center of the cluster. Entropic effects at CNT growth temperatures may dominate over these small energy differences.

Table 1 Energies, in $\mathrm{eV}$, of $\mathrm{CrCO}_{54}$ clusters when the $\mathrm{Cr}$ atom is at an edge and apex position in the outer layer, when it is in the subsurface layer and when it is the center atom. The optimised geometries and energies are obtained using GGA spin polarised and LDA spin polarised and non spin polarised methods

\begin{tabular}{c|c|c|c|c}
\hline \multirow{2}{*}{ Method } & \multicolumn{4}{|c}{ Energy (eV) } \\
\cline { 2 - 5 } & Edge & Apex & Subsurface & center \\
\hline GGA spin polarised & -334.7 & -334.7 & -334.3 & -334.9 \\
\hline LDA spin polarised & -380.6 & -380.6 & -380.9 & -380.9 \\
\hline LDA non spin polarised & -367.7 & -367.4 & -368.5 & -367.2 \\
\hline
\end{tabular}

Table 2 shows the energies of $\mathrm{Cr}_{5} \mathrm{Co}_{50}$ clusters when the five $\mathrm{Cr}$ atoms are placed in either the outer or subsurface layers. All of the methods show that, at $0 \mathrm{~K}$, the $\mathrm{Cr}$ atoms prefer to be in the subsurface layer. The energy difference between the structure where the $\mathrm{Cr}$ atoms are in the outer and subsurface layers is rather large (2-3 eV for the spin polarised methods and $5 \mathrm{eV}$ for the non spin polarised method), so that this may dominate over the entropic contributions even at the high temperatures used in CNT growth.

\section{瀷 Springer}


Table 2 Energies, in $\mathrm{eV}$, of $\mathrm{Cr}_{5} \mathrm{CO}_{50}$ clusters when the $\mathrm{Cr}$ atoms are in the outer or subsurface layers. The optimised geometries and energies are obtained using GGA spin polarised and LDA spin polarised and non spin polarised methods

\begin{tabular}{c|c|c}
\hline \multirow{2}{*}{ Method } & \multicolumn{2}{|c}{ Energy $(\mathrm{eV})$} \\
\cline { 2 - 3 } & Outer & Subsurface \\
\hline GGA spin polarised & -339.8 & -342.3 \\
\hline LDA spin polarised & -388.3 & -390.4 \\
\hline LDA non spin polarised & -377.5 & -382.4 \\
\hline
\end{tabular}

The validity of the $0 \mathrm{~K}$ results at high temperatures was investigated using direct dynamics simulations of the $\mathrm{Cr}_{5} \mathrm{Co}_{50}$ clusters at $2000 \mathrm{~K}$. Due to the computational expense of the spin polarised methods, and since the spin polarised and non spin polarised methods are in qualitative agreement, the direct dynamics were based on LDA non spin polarised forces. Computational time required for each trajectory ( 4000 integration steps and using 16 processors) were typically in excess of one month. Figure 6 shows the change in the center of mass separation for each of the five $\mathrm{Cr}$ atoms when they are initially in the outer layer (Fig. 6(a)) or in the subsurface layer (Fig. 6(b)). The blue line shows the change in the Co center of mass separation averaged over all 50 atoms. It is clear that, in agreement with the $0 \mathrm{~K}$ calculations, the $\mathrm{Cr}$ atoms do not prefer the outer layer sites and penetrate and remain in the cluster.

To shed further light on the effect of the high temperature on the cluster composition, we calculated the (LDA non spin polarised) energies of the final structures obtained in the two simulations shown in Fig. 6, as well as the energies after geometry optimisation of these structures. These energies can be compared to the energies of the initial structures shown in Table 2, i.e., -377.5 and $-382.4 \mathrm{eV}$ when the $\mathrm{Cr}$ atoms are in the outer and subsurface layers, respectively. The energies of the final structures obtained in the two simulations are $-360.8 \mathrm{eV}$ and $-360.2 \mathrm{eV}$, respectively, and after geometry optimisation they are -378.5 and -377.7 , respectively. As expected, the alloy structures and energies at the end of the two simulations are similar. Of interest is that the high temperature used in the simulations $(2000 \mathrm{~K})$ allows the cluster to sample geometries that are more than $15 \mathrm{eV}$ above the $0 \mathrm{~K}$ optimised structures. In spite of these large entropic contributions the trends of the $0 \mathrm{~K}$ calculations are also valid at $2000 \mathrm{~K}$.

Further work is required to ascertain if other alloy systems show the same trends, i.e., if trends in the distribution of alloy components obtained from calculations at $0 \mathrm{~K}$ are valid at high temperatures. These tests should include systems where one of the alloy components prefers to be located in the outer layer at $0 \mathrm{~K}$, but may penetrate into the cluster and mix with the second component at high temperatures (due to entropy of mixing). If certain systems show this behaviour then it would be of interest (and of relevance to applications) to determine the minimum

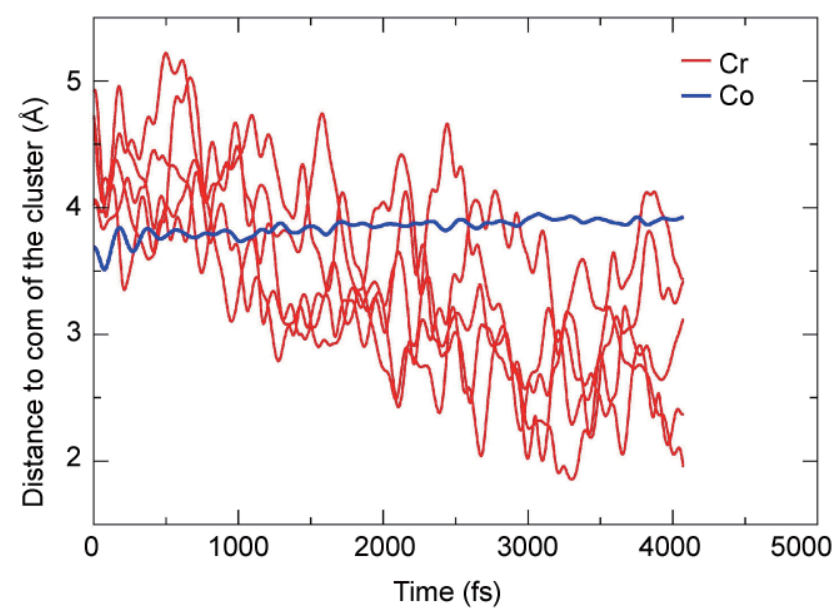

(a)

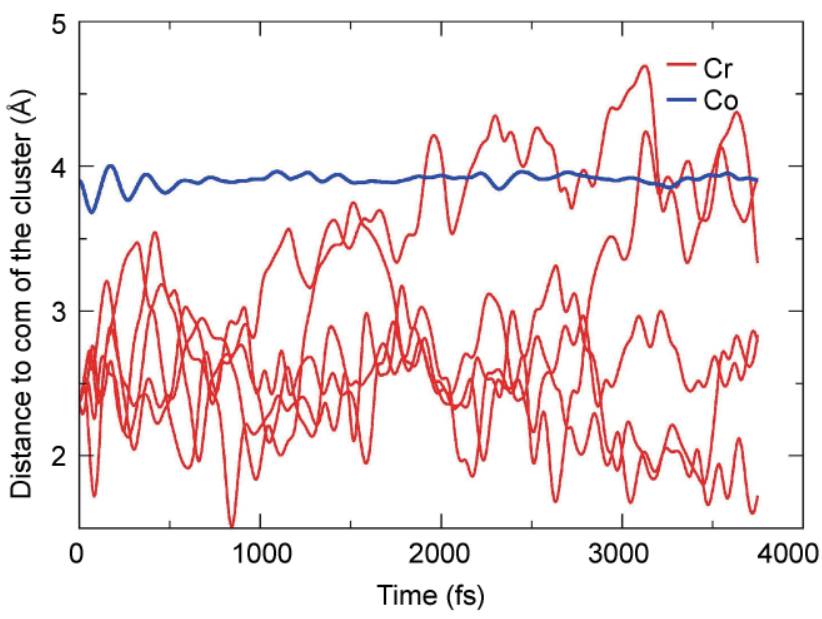

(b)

Figure 6 Change in the center of mass (com) separation of the $\mathrm{Cr}$ atoms (red) in the $\mathrm{Cr}_{5} \mathrm{CO}_{50}$ clusters at $2000 \mathrm{~K}$, when they are initially in the outer (a) and subsurface layers (b). The blue curve shows the change in the Co center of mass separation averaged over all 50 atoms 
temperature at which the $0 \mathrm{~K}$ structure is no longer valid. In addition, the effect of adding other species (such as carbon) to the alloy system, or attaching structures (such as CNTs) onto the cluster, needs to be investigated.

\section{Conclusions}

TBMC has been used to study SWNT-Ni cluster systems of relevance to SWNT growth and regrowth. $\mathrm{Ni}$ atoms that are initially on the surface of the SWNT or that are clustered near the SWNT end diffuse to the nanotube end so that virtually none of the $\mathrm{Ni}$ atoms are located inside the nanotube. This occurs, in part, due to the lowering of the $\mathrm{Ni}$ atom energies when they retract from the SWNT and are included in the interior of the cluster. The chirality of the SWNT is not changed when the atoms aggregate at the SWNT end, even when some of the SWNT end atoms dissolve into the cluster. This indicates that SWNT regrowth is a potential route for chiralitycontrolled SWNT production.

DFT-based geometry optimisation and direct dynamics at $2000 \mathrm{~K}$ show that $\mathrm{Cr}$ and Mo atoms in $\mathrm{Cr}_{5} \mathrm{Co}_{50}$ and $\mathrm{Mo}_{5} \mathrm{Co}_{50}$ clusters prefer to be distributed in the interior of the clusters. This indicates that, at least for these bimetallic systems, the computationally cheaper optimisation methods predict trends in alloy metal cluster compositions that are also relevant for temperatures as high as $2000 \mathrm{~K}$. Further calculations on other systems are needed to reveal if this is typical for metallic alloys or, if this is not the case, the temperatures at which the $0 \mathrm{~K}$ optimisation results are no longer valid. Further calculations will also reveal the effect that adsorbants, or other structures that are adhered to the cluster surface (such as SWNTs), have on the distribution of the atoms in the cluster.

\section{Acknowledgements}

The calculations were performed on C3SE computing resources and the Swedish National Supercomputing facilities. Financial support was obtained from the Swedish Research Council, the Swedish Foundation for Strategic Research (CARAMEL consortium), and
University of Gothenburg Nanoparticle Platform. We thank Francóis Ducastelle for fruitful discussions.

\section{References}

[1] Reich, S.; Thomsen, C.; Maultzch, J. Carbon Nanotubes: Basic Concepts and Physical Properties; Wiley-VCH: Weinheim, 2004.

[2] Dresselhaus, G.; Avouris, P. Carbon Nanotubes: Synthesis, Structure, Properties and Applications; Springer: Berlin, 2001.

[3] Bachilo, S. M.; Balzano, L.; Herrera, J. E.; Pompeo, F.; Resasco, D. E.; Weisman, R. B. Narrow $(n, m)$-distribution of single-walled carbon nanotubes grown using a solid supported catalyst. J. Am. Chem. Soc. 2003, 125, 11186 $-11187$

[4] Wang, Y. H.; Kim, M. J.; Shan, H.; Kittrell, C.; Fan, H.; Ericson, L.; Hwang, W. -F.; Arepalli, S.; Hauge, R. H.; Smalley, R. E. Continued growth of single-walled carbon nanotubes. Nano Lett. 2005, 5, 997-1002.

[5] Abild-Pedersen, F.; Nørskov, J. K.; Rostrup-Nielsen, J. R.; Sehested J.; Helveg, S. Mechanisms for catalytic carbon nanofiber growth studied by ab initio density functional theory calculations. Phys. Rev. B 2006, 73, 115419.

[6] Ding, F.; Larsson, P.; Larsson, J. A.; Ahuja, R.; Duan, H.; Rosén, A.; Bolton, K. The importance of strong carbonmetal adhesion for catalytic nucleation of single-walled carbon nanotubes. Nano Lett. 2008, 8, 463-468.

[7] Shibuta, Y.; Maruyama, S. Molecular dynamics simulation of generation process of SWNTs. Physica B 2002, 323, 187-189.

[8] Ding, F.; Bolton, K.; Rosén, A. Nucleation and growth of single-walled carbon nanotubes: A molecular dynamics study. J. Phys. Chem. B. 2004, 108, 17369-17377.

[9] Zheng, G.; Irle, S.; Morokuma, K. Fe/C interactions during SWNT growth with $C_{2}$ feedstock molecules: $A$ quantum chemical molecular dynamics study. J. Nanosci. Nanotechnol. 2006, 6, 1259-1270.

[10] Gavillet, J.; Loiseau, A.; Journet, C.; Willaime, F.; Ducastelle, F.; Charlier, J. -C. Root-growth mechanism for single-wall carbon nanotubes. Phys. Rev. Lett. 2001, 87, 275504.

[11] Raty, J. -Y.; Gygi, F.; Galli, G. Growth of carbon nanotubes on metal nanoparticles: A microscopic mechanism from ab initio molecular dynamics simulations. Phys. Rev. Lett. 2005, 95, 96103. 
[12] Amara, H.; Roussel, J. -M.; Bichara, C.; Gaspard, J. -P.; Ducastelle, F. Tight-binding potential for atomistic simulations of carbon interacting with transition metals: Application to the Ni-C system. Phys. Rev. B 2009, 79, 014109.

[13] Amara, H.; Bichara, C.; Ducastelle, F. Understanding the nucleation mechanisms of carbon nanotubes in catalytic chemical vapour deposition. Phys. Rev. Lett. 2008, 100, 056105.

[14] Börjesson, A.; Zhu, W.; Amara, H.; Bichara, C.; Bolton, $K$. Computational studies of metal-carbon nanotube interfaces for regrowth and electronic transport. Nano Letters, 2009, 9, 1117-1120.

[15] Frenkel, D.; Smit, B. Understanding Molecular Simulation; Oxford University Press: Oxford, 1997.

[16] Kresse, G.; Furthmuller, J. Efficient iterative schemes for ab initio total-energy calculations using a plane-wave basis set. J. Phys. Rev. B 1996, 54, 11169-11186.

[17] Perdew, J. P.; Chevary, J. A.; Vosko, S. H.; Jackson, K. A.; Pederson, M. R.; Singh, D. J.; Fiolhais, C. Atoms, molecules, solids and surfaces: Applications of the generalized gradient approximation for exchange and correlation. Phys. Rev. B 1992, 46, 6671-6687.

[18] Perdew, J. P.; Zunger, A. Self-interaction correction to density-functional approximations for many-electron systems. Phys. Rev. B 1981, 23, 5048-5079

[19] Ceperley, D. M.; Alder, B. J. Ground state of the electron gas by a stochastic method. Phys. Rev. Lett. 1980, 45, 566-569

[20] Schebarchov, D.; Hendy, S. C. Capillary absorption of metal nanodroplets by single-wall carbon nanotubes. Nano Lett. 2008, 8, 2253-2257. 